\title{
Hydrophobic Waters in Bromodomains ${ }^{\dagger}$
}

\author{
Serena G. Piticchio 1,*, Miriam Martínez-Cartró ${ }^{1}$, Salvatore Scaffidi ${ }^{1}$, Sergio Rodríguez-Arévalo ${ }^{2}$, \\ Andrea Bagán ${ }^{2}$, Ainoa Sánchez-Arfelis ${ }^{2}$, Carmen Escolano ${ }^{2}$, Carles Galdeano ${ }^{1}$ and Xavier Barril ${ }^{1}$ \\ 1 Department of Pharmacy, Pharmaceutical Technology and Physical Chemistry, Faculty of Pharmacy and \\ Food Sciences, University of Barcelona, Institute of Biomedicine of the University of Barcelona (IBUB), \\ and Catalan Institution for Research and Advanced Studies (ICREA), 08028 Barcelona, Spain \\ 2 Laboratory of Medicinal Chemistry (Associated Unit to CSIC), Department of Pharmacology, \\ Toxicology and Therapeutic Chemistry, Faculty of Pharmacy and Food Sciences, \\ and Institute of Biomedicine of the University of Barcelona (IBUB), 08028 Barcelona, Spain \\ * Correspondence: serena_piticchio@ub.edu \\ + Presented at the 2nd Molecules Medicinal Chemistry Symposium (MMCS): Facing Novel Challenges in \\ Drug Discovery, Barcelona, Spain, 15-17 May 2019.
}

Published: 16 August 2019

Keywords: bromodomains; BRD4; structural water molecules; fragment-based drug design; medicinal chemistry; crystallography; drug discovery

Targeting epigenetic proteins is a rapidly growing area for medicinal chemistry and drug discovery. Recent years have seen an explosion of interest in developing small molecules binding to bromodomains due to their implication in cancer, inflammation, and a plethora of diseases. Several small-molecule inhibitors and degraders that target bromodomains have entered the clinic, and many more are increasingly being used as chemical probes to describe bromodomain biology. From a structural point of view, crystallographic studies of bromodomains describe, as a common feature, five water molecules as an integral part of the acetyl-lysine binding pocket. These water molecules are essential in druggability and are described as a functional part of the protein [1,2].

In this framework, we focused our attention on the description of the hydrophilic/hydrophobic character of these molecules, which seem to create a favorable environment for the recognition of hydrophobic groups. To this end, and following fragment-based drug design techniques, here we describe a new family of small molecules with a 5-phenylthiazolo[2,3-c][1,2,4]triazol nucleus and probe the water site with various substituents at the 3-position endowing hydrophilic or hydrophobic properties. In this work, we present the theoretical calculations, the synthesis of the new compounds, the results of differential scanning fluorimetry (DSF) and isothermal titration calorimetry (ITC), and the crystal structures of three of our compounds with the target protein. The study sheds light on the counterintuitive behavior of the water molecules in this particular environment.

\section{References}

1. Galdeano, C.; Ciulli, A. Selectivity on-target of bromodomain chemical probes by structure-guided medicinal chemistry and chemical biology. Future Med. Chem. 2016, 8, 1655-1680.

2. Zhang, X.; Chen, K.; Wu, Y.-D.; Wiest, O. Protein dynamics and structural waters in bromodomains. PLoS ONE 2017, 12, e0186570.

(C) 2019 by the authors. Licensee MDPI, Basel, Switzerland. This article is an open access article distributed under the terms and conditions of the Creative Commons Attribution (CC BY) license (http://creativecommons.org/licenses/by/4.0/). 